\title{
Some Characterizations of Exponential Distribution
}

\author{
M. Ahsanullah ${ }^{1} \&$ M. Z. Anis ${ }^{2}$ \\ ${ }^{1}$ Rider University, Lawrenceville, NJ 08648, USA \\ ${ }^{2}$ SQC \& OR Unit, Indian Statistical Institute, 203, B T Road, Calcutta 700 108, India \\ Correspondence: M. Ahsanullah, Rider University, Lawrenceville, NJ 08648, USA. E-mail: ahsan@ $@$ rider.edu \\ Received: April 11, 2017 Accepted: May 2, 2017 Online Published: August 24, 2017 \\ doi:10.5539/ijsp.v6n5p132 URL: https://doi.org/10.5539/ijsp.v6n5p132
}

\begin{abstract}
There are some characterizations of the exponential distribution based on the relation of the maximum of two observations expressed as linear combination of the two observations. In this paper some generalizations of this known characterization of the exponential distribution using the relations between the maximum and minimum of $n(\geq 2)$ independent and identically distributed random variables having absolutely continuous (with respect to Lebesgue measure) distribution function will be presented.
\end{abstract}

Mathematical Subject Classification (2010): 60E05; 62E10; 62H10.

Keywords: Hazard rate; identical distributions, order statistics, random variables

\section{Introduction}

Many times the researcher wants to verify whether the data that she/he has obtained belong to a certain family of distribution. For that purpose, the researcher has to rely on the characterization of the assumed distribution. Hence the characterization of distributions becomes important and essential. The univariate exponential distribution is the most commonly used distribution in modeling reliability and life testing analysis. There are many characterizations of the exponential distribution using ordered random variables. Ferguson (1967) characterized the exponential distribution using the regression properties of the first two order statistics. There are many generalizations of this characterization by using order statistics and record values. For details, see Ahsanullah et al. (2013) and David and Nagaraja (2009). Recently Arnold and Villasenor (2013) characterized the exponential distribution by using the identical distribution of $X_{1}+\frac{X_{2}}{2}$ and $\max \left(X_{1}, X_{2}\right)$ assuming $X_{1}$ and $X_{2}$ as identically distributed absolutely continuous (with respect to Lebesgue measure) non-negative random variables with the restriction that their distribution function is infinitely differentiable. In this paper some generalizations of this characterization of the exponential distribution based on the relation between maximum and minimum of $n(\geq 2)$ independent and identically distributed continuous random variables are derived.

We say a random variable $X$ has the exponential distribution if its probability density function (pdf) $f(x ; \lambda)$ is given by $f(x ; \lambda)=\lambda e^{-\lambda x}, \lambda>0 ; x \geq 0$.

\section{The Main Results}

We shall present two interesting characterizations in this paper. To prove the first characterization, we shall need the following lemma.

Lemma 2.1: Suppose that the random variable $X$ has an absolutely continuous (with respect to Lebesgue measure) cumulative distribution function (cdf) $F(x)$ with $F(0)=0$ and $F(x)>0$ for all $x>0$. Let the associated probability distribution function (pdf) be denoted by $f(x)$. Assume that $F(x)$ is infinitely differentiable. Let $H(x)=F(x) f(x)$. Then

if

$$
H^{(k)}(0)=(-1)^{k-1}\left(2^{k}-1\right)\{f(0)\}^{k+1}, \quad k=1,2, \cdots,
$$

$$
f^{(r)}(0)=(-1)^{r}\{f(0)\}^{r+1}, \quad r=1,2, \cdots, k-1
$$




\section{Proof:}

We shall prove by induction.

Let us define $f^{(0)}(x)=f(x)$.

Since $H(x)=F(x) f(x)$, we have $H(0)=0$. Routine differentiation with respect to $x$ and simplifying yields the following results:

$$
\begin{aligned}
H^{(1)}(0) & =F(x) f^{(1)}(x)+\left.(f(x))^{2}\right|_{x=0}=\{f(0)\}^{2} ; \\
\mathrm{H}^{(2)}(0) & =\mathrm{F}(\mathrm{x}) \mathrm{f}^{(2)}(\mathrm{x})+2 \mathrm{f}(\mathrm{x}) \mathrm{f}^{(1)}(\mathrm{x})+\left.\mathrm{f}(\mathrm{x}) \mathrm{f}^{(1)}(\mathrm{x})\right|_{\mathrm{x}=0} \\
& =3 \mathrm{f}(0) \mathrm{f}^{(1)}(0) \\
& =(-1)\left(2^{2}-1\right)\{\mathrm{f}(0)\}^{3} \text { if } \mathrm{f}^{(1)}(0)=-\{\mathrm{f}(0)\}^{2} .
\end{aligned}
$$

Similarly, we have

$$
\begin{aligned}
H^{(3)}(0) & =F(x) f^{(3)}(x)+3 f(x) f^{(2)}(x)+3 f^{(1)}(x) f^{(1)}(x)+\left.f^{(2)}(x) f(x)\right|_{x=0} \\
& =4 f(0) f^{(2)}(0)+3\left\{f^{(1)}(0)\right\}^{2} \\
& =(-1)^{2}\left(2^{3}-1\right)\{f(0)\}^{4} \text { if } f^{(r)}(0)=(-1)^{r}\{f(0)\}^{r+1}, \quad r=1,2 .
\end{aligned}
$$

It is easy to see that we can write

$$
\mathrm{H}^{(\mathrm{k})}(\mathrm{x})=\mathrm{F}(\mathrm{x}) \mathrm{f}^{(\mathrm{k})}(\mathrm{x})+\sum_{\mathrm{r}=1}^{\mathrm{k}}\left(\begin{array}{l}
\mathrm{k} \\
\mathrm{r}
\end{array}\right) \mathrm{f}^{(\mathrm{r}-1)}(\mathrm{x}) \mathrm{f}^{(\mathrm{k}-\mathrm{r})}(\mathrm{x}) .
$$

Then, we have

$$
\begin{aligned}
\mathrm{H}^{(\mathrm{k})}(0) & =\sum_{\mathrm{r}=1}^{\mathrm{k}}\left(\begin{array}{l}
\mathrm{k} \\
\mathrm{r}
\end{array}\right) \mathrm{f}^{(\mathrm{r}-1)}(0) \mathrm{f}^{(\mathrm{k}-\mathrm{r})}(0) \\
& =\mathrm{kf}(0) \mathrm{f}^{(\mathrm{k}-1)}(0)+\mathrm{f}^{(\mathrm{k}-1)}(0) \mathrm{f}(0)+\sum_{\mathrm{r}=2}^{\mathrm{k}-1}\left(\begin{array}{l}
\mathrm{k} \\
\mathrm{r}
\end{array}\right) \mathrm{f}^{(\mathrm{r}-1)}(0) \mathrm{f}^{(\mathrm{k}-\mathrm{r})}(0) \\
& =(\mathrm{k}+1) \mathrm{f}(0) \mathrm{f}^{(\mathrm{k}-1)}(0)+\sum_{\mathrm{r}=2}^{\mathrm{k}-1}\left(\begin{array}{l}
\mathrm{k} \\
\mathrm{r}
\end{array}\right)(-1)^{\mathrm{r}-1}\{\mathrm{f}(0)\}^{\mathrm{r}}(-1)^{\mathrm{k}-\mathrm{r}}\{\mathrm{f}(0)\}^{\mathrm{k}-\mathrm{r}+1} \\
& =(\mathrm{k}+1) \mathrm{f}(0)(-1)^{\mathrm{k}-1}\{\mathrm{f}(0)\}^{\mathrm{k}}+\sum_{\mathrm{r}=2}^{\mathrm{k}-1}\left(\begin{array}{l}
\mathrm{k} \\
\mathrm{r}
\end{array}\right)(-1)^{\mathrm{k}-1}\{\mathrm{f}(0)\}^{\mathrm{k}+1} \\
& =(-1)^{\mathrm{k}-1}(\mathrm{k}+1)\{\mathrm{f}(0)\}^{\mathrm{k}+1}+(-1)^{\mathrm{k}-1}\{\mathrm{f}(0)\}^{\mathrm{k}+1} \sum_{\mathrm{r}=2}^{\mathrm{k}-1}\left(\begin{array}{l}
\mathrm{k} \\
\mathrm{r}
\end{array}\right) \\
& =(-1)^{\mathrm{k}-1}\{\mathrm{f}(0)\}^{\mathrm{k}+1}\left[\mathrm{k}+1+2^{\mathrm{k}}-\left(\begin{array}{l}
\mathrm{k} \\
0
\end{array}\right)-\left(\begin{array}{l}
\mathrm{k} \\
1
\end{array}\right)-\left(\begin{array}{l}
\mathrm{k} \\
\mathrm{k}
\end{array}\right)\right] \\
& =(-1)^{\mathrm{k}-1}\{\mathrm{f}(0)\}^{\mathrm{k}+1}\left[\mathrm{k}+1+2^{\mathrm{k}}-1-\mathrm{k}-1\right] \\
& =(-1)^{\mathrm{k}-1}\{\mathrm{f}(0)\}^{\mathrm{k}+1}\left(2^{\mathrm{k}}-1\right)
\end{aligned}
$$


This completes the proof of Lemma 2.1 .

Theorem 2.1. Suppose $X, X_{1}$ and $X_{2}$ are independent and identically distributed random variables with cumulative distribution function (cdf) $F(x)$ with $F(0)=0$ and $F(0)>0$ for all $x>0$. Assume that $F(x)$ is absolutely continuous (with respect to Lebesgue measure) and infinitely differentiable with $\frac{d F(x)}{d x}=f(x)$ and $f(0)>0$. Let $\mathrm{Z}=\max \left(\mathrm{X}_{1}, \mathrm{X}_{2}\right)$ and $\mathrm{W}=\min \left(\mathrm{X}_{1}, \mathrm{X}_{2}\right)$. Then $\mathrm{Z}={ }^{\mathrm{d}} \mathrm{W}+\mathrm{X}$, where $=^{\mathrm{d}}$ denotes equality in distribution and $\mathrm{X}$ is independent of $W$, if and only if $F(x)=1-e^{-\lambda x}, x \geq 0$ and $\lambda$ is an arbitrary positive real number.

\section{Proof:}

Necessity: Assume that $F(x)=1-e^{-\lambda x}, x \geq 0, \lambda>0$. Let $f_{1}(x)$ and $f_{2}(x)$ be the pdfs of $Z$ and $W+X$ respectively. Then it is easy to see that

$$
f_{1}(x)=2 F(x) f(x)=2 \lambda\left(1-e^{-\lambda x}\right) e^{-\lambda x}
$$

and

$$
\begin{aligned}
f_{2}(x) & =\int_{0}^{x} 2[1-F(x-y)][f(x-y)] f(y) d y \\
& =\int_{0}^{x} 2 \lambda^{2} e^{-\lambda(x-y)} e^{-\lambda(x-y)} e^{-\lambda y} d y \\
& =2 \lambda e^{-\lambda x}\left(1-e^{-\lambda x}\right)
\end{aligned}
$$

Thus, $Z={ }^{\mathrm{d}} \mathrm{W}+\mathrm{X}$.

Sufficiency: Suppose $f_{1}(x)=f_{2}(x)$ for all $x \geq 0$. Then, we can write

$$
\begin{aligned}
& f_{2}(x)=\int_{0}^{x} 2[1-F(x-y)][f(x-y)] f(y) d y \\
= & \int_{0}^{x} 2 f(x-y) f(y) d y-2 \int_{0}^{x} F(x-y) f(x-y) f(y) d y \\
= & 2 \int_{0}^{x} f(x-y) f(y) d y-2 \int_{0}^{x} H(x-y) f(y) d y,
\end{aligned}
$$

Since $H(x)=F(x) f(x)$.

Since $f_{1}(x)=2 H(x)$, equating $f_{1}(x)$ and $f_{2}(x)$, we obtain on simplification

$$
\int_{0}^{x} f(x-y) f(y) d y=H(x)+\int_{0}^{x} H(x-y) f(y) d y .
$$

Differentiating twice both sides of (2.3) with respect to $\mathrm{x}$ and putting $\mathrm{x}=0$, we have

$$
2 f(0) f^{(1)}(0)=H^{(2)}(0)+f(0) H^{(1)}(0) \text {. }
$$

From Lemma 1, we have,

and

$$
\begin{gathered}
H(0)=0 ; \\
H^{(1)}(0)=\{f(0)\}^{2},
\end{gathered}
$$

$$
H^{(2)}(0)=3 f(0) f^{(1)}(0)
$$

Thus, 


$$
2 f(0) f^{(1)}(0)=3 f(0) f^{(1)}(0)+f(0)[f(0)]^{2},
$$

i.e.

$$
\mathrm{f}^{(1)}(0)=-[\mathrm{f}(0)]^{2}
$$

Similarly differentiating thrice both sides of (2.3) with respect to $\mathrm{x}$ and putting $\mathrm{x}=0$, we have

$$
f(0) f^{(2)}(0)+\left[f^{(1)}(0)\right]^{2}+f(0) f^{(2)}(0)=H^{(3)}(0)+f(0) H^{(2)}(0)+f^{(1)}(0) H^{(1)}(0) .
$$

As before, using Lemma 1 and simplifying, we obtain

$$
f^{(2)}(0)=[f(0)]^{3}
$$

Suppose that

$$
f^{(r)}(0)=(-1)^{r}\{f(0)\}^{r+1}, \quad r=1,2, \cdots, k-1 .
$$

On differentiating $(\mathrm{k}+2)$ times both sides of $(2.3)$ with respect to $x$ and putting $x=0$, we obtain

$$
\begin{gathered}
\sum_{r=0}^{k} f^{(r)}(0) f^{(k+1-r)}(0)+f(0) f^{(k+1)}(0) \\
=H^{(k+2)}(0)+f(0) H^{(k+1)}(0)+\sum_{r=1}^{k} f^{(r)}(0) H^{(k+1-r)}(0)
\end{gathered}
$$

The LHS of Eqn 2.4 is given by

$$
\begin{aligned}
& =\quad \sum_{r=0}^{k} f^{(r)}(0) f^{(k+1-r)}(0)+f(0) f^{(k+1)}(0) \\
& =\quad 2 f(0) f^{(k+1)}(0)+\sum_{r=0}^{k} f^{(r)}(0) f^{(k+1-r)}(0) \\
& =\quad 2 f(0) f^{(k+1)}(0)+\sum_{r=1}^{k} f^{(r)}(0)(-1)^{k+1-r}[f(0)]^{k+2-r} \\
& =\quad 2 f(0) f^{(k+1)}(0)+(-1)^{k+1}[f(0)]^{k+2} \sum_{r=1}^{k} f^{(r)}(0)(-1)^{-r}[f(0)]^{-r} \\
& =\quad 2 f(0) f^{(k+1)}(0)+(-1)^{k+1}[f(0)]^{k+2} \sum_{r=1}^{k}(-1)^{r}[f(0)]^{r+1}(-1)^{-r}[f(0)]^{-r} \\
& =\quad 2 f(0) f^{(k+1)}(0)+(-1)^{k+1}[f(0)]^{k+2} \sum_{r=1}^{k} f(0) \\
& =2 f(0) f^{(k+1)}(0)+(-1)^{k+1} k[f(0)]^{k+3}
\end{aligned}
$$

The RHS of Eqn 2.4 is given by 


$$
\begin{aligned}
& =\quad H^{(k+2)}(0)+f(0) H^{(k+1)}(0)+\sum_{r=1}^{k} f^{(r)}(0) H^{(k+1-r)}(0) \\
& =\mathrm{H}^{(\mathrm{k}+2)}(0)+\sum_{\mathrm{r}=0}^{\mathrm{k}} \mathrm{f}^{(\mathrm{r})}(0) \mathrm{H}^{(\mathrm{k}+1-\mathrm{r})}(0) \\
& =\quad H^{(k+2)}(0)+\sum_{r=0}^{k} f^{(r)}(0)(-1)^{k-r}[f(0)]^{k+2-r}\left(2^{k+1-r}-1\right) \\
& =\quad H^{(k+2)}(0)+\sum_{r=0}^{k}(-1)^{r}[f(0)]^{r+1}(-1)^{k-r}[f(0)]^{k+2-r}\left(2^{k+1-r}-1\right) \\
& =H^{(k+2)}(0)+\sum_{r=0}^{k}(-1)^{k}[f(0)]^{k+3}\left(2^{k+1-r}-1\right) \\
& =\quad \mathrm{H}^{(\mathrm{k}+2)}(0)+(-1)^{\mathrm{k}}[\mathrm{f}(0)]^{\mathrm{k}+3} \sum_{\mathrm{r}=0}^{\mathrm{k}}\left(2^{\mathrm{k}+1-\mathrm{r}}-1\right) \\
& =(-1)^{\mathrm{k}+1}[\mathrm{f}(0)]^{\mathrm{k}+3}\left(2^{\mathrm{k}+2 \mathrm{r}}-1\right)+(-1)^{\mathrm{k}}[\mathrm{f}(0)]^{\mathrm{k}+3}\left[2\left(2^{\mathrm{k}+1}-1\right)-(\mathrm{k}+1)\right] \\
& =(-1)^{\mathrm{k}}[\mathrm{f}(0)]^{\mathrm{k}+3}\left\{-1\left(2^{\mathrm{k}+2}-1\right)+2^{\mathrm{k}+2}-2-\mathrm{k}-1\right\} \\
& =(-1)^{\mathrm{k}}[\mathrm{f}(0)]^{\mathrm{k}+3}\{-2-\mathrm{k}\} \\
& =(-1)^{\mathrm{k}+1}[\mathrm{f}(0)]^{\mathrm{k}+3}\{2+\mathrm{k}\}
\end{aligned}
$$

Equating both sides we get

$$
2 f(0) f^{(k+1)}(0)+(-1)^{k+1} k[f(0)]^{k+3}=(-1)^{k+1}[f(0)]^{k+3}\{2+k\}
$$

Or

$$
2 \mathrm{f}(0) \mathrm{f}^{(\mathrm{k}+1)}(0)=(-1)^{\mathrm{k}+1}[\mathrm{f}(0)]^{\mathrm{k}+3}\{2+\mathrm{k}-\mathrm{k}\}=2(-1)^{\mathrm{k}+1}[\mathrm{f}(0)]^{\mathrm{k}+3}
$$

Hence,

$$
\mathrm{f}(0) \mathrm{f}^{(\mathrm{k}+1)}(0)=(-1)^{\mathrm{k}+1}[\mathrm{f}(0)]^{\mathrm{k}+3}
$$

Or equivalently,

$$
\mathrm{f}^{(\mathrm{k}+1)}(0)=(-1)^{\mathrm{k}+1}[\mathrm{f}(0)]^{\mathrm{k}+2}
$$

Repeating the same procedure, we obtain

$$
\mathrm{f}^{(\mathrm{m})}(0)=(-1)^{\mathrm{m}}[\mathrm{f}(0)]^{\mathrm{m}+1} ; \mathrm{m}>\mathrm{k}+1
$$

Hence,

$$
f^{(j)}(0)=(-1)^{j}[f(0)]^{j+1} ; j=1,2, \cdots .
$$

Expanding $f(x)$ in Taylor series, we have

$$
f(x)=f(0)+\frac{f^{(1)}(0)}{1 !} x+\frac{f^{(2)}(0)}{2 !} x^{2}+\frac{f^{(3)}(0)}{3 !} x^{3}+\cdots
$$




$$
\begin{aligned}
& =f(0)-\frac{[f(0)]^{2}}{1 !} x+\frac{[f(0)]^{3}}{2 !} x^{2}-\frac{[f(0)]^{4}}{3 !} x^{3}+\cdots \\
& =f(0) e^{-x f(0)}
\end{aligned}
$$

Thus,

$$
f(x)=\lambda e^{-\lambda x} .
$$

This completes the proof of Theorem 2.1.

Remark 2.1: In Theorem 2.1 above, the equality of distribution can be replaced by the equality in expectation.

We define the hazard rate $h(x)=\frac{f(x)}{1-F(x)}$ for $0<F(x)<1$. We shall say that $F(x) \in \mathcal{E}$ if $h(x)$ is either non-decreasing or non-increasing with respect to $x$.

The following theorem characterizes the exponential distribution using the distributional relation between the maximum and minimum of $n(>2)$ random variables.

Theorem 2.2. Suppose $X_{1}, X_{2}, \cdots, X_{n}$ are $n$ independent and identically distributed random variables with absolutely continuous (with respect to Lebesgue measure) cumulative distribution function (cdf) $F(x)$ with $F(0)=0$ and $F(x)>0$ for all $x>0$. Let the corresponding probability density function (pdf) be denoted by $f(x)$. Let $Z_{n}=$ $\max \left(X_{1}, X_{2}, \cdots, X_{n}\right), W_{n}=\min \left(X_{1}, X_{2}, \cdots, X_{n}\right)$, and $X_{i, n}, i=1,2, \cdots, n$ be the $i$ th order statistic. Then the following two conditions are identical:

a) $\mathrm{F}(\mathrm{x})=1-\mathrm{e}^{-\lambda \mathrm{x}}, \mathrm{x} \geq 0, \lambda>0$;

b) $Z_{n}-W_{n}=^{d} X_{n-1, n-1}$ and $F(x) \in \mathcal{E}$.

Proof:

(a) $\Rightarrow$ (b)

It is known (see, for example Ahsanullah and Nevzorov (2005) page 51; Arnold et al. (1992) page 72) that

$$
X_{i, n}={ }^{d} \frac{X_{1}}{n}+\frac{X_{2}}{n-1}+\cdots+\frac{X_{i}}{n-i+1}, \quad i=1,2, \cdots, n .
$$

Hence we have

$$
X_{n, n}={ }^{d} \frac{X_{1}}{n}+\frac{X_{2}}{n-1}+\cdots+\frac{X_{n}}{1}
$$

and

$$
\mathrm{X}_{1, \mathrm{n}}={ }^{\mathrm{d}} \frac{\mathrm{X}_{1}}{\mathrm{n}}
$$

Therefore,

$$
X_{n, n}-X_{1, n}=\frac{X_{2}}{n-1}+\cdots+\frac{X_{n}}{1}={ }^{d} X_{n-1, n-1}
$$

(b) $\Rightarrow$ (a)

The pdf of $f_{Z, W}(x)$ of $Z_{n}-W_{n}$ is given by

$$
f_{\mathrm{Z}, W}(x)=\int_{0}^{\infty} n(n-1)[F(x+y)-F(y)]^{n-2} f(x+y) f(y) d y
$$

It is easy to show (see e.g. Arnold et al. (1992) page 31) that the corresponding cdf $F_{Z, W}(x)$ of $Z_{n}-W_{n}$ is given by

$$
\mathrm{F}_{\mathrm{Z}, \mathrm{W}}(\mathrm{x})=\int_{0}^{\infty} \mathrm{n}[\mathrm{F}(\mathrm{x}+\mathrm{y})-\mathrm{F}(\mathrm{y})]^{\mathrm{n}-1} \mathrm{f}(\mathrm{y}) \mathrm{dy}=\int_{0}^{\infty} \mathrm{n}[\overline{\mathrm{F}}(\mathrm{x}+\mathrm{y})-\overline{\mathrm{F}}(\mathrm{y})]^{\mathrm{n}-1} \mathrm{f}(\mathrm{y}) d y, \text { where } \overline{\mathrm{F}}(\mathrm{x})=1-\mathrm{F}(\mathrm{x})
$$

The pdf $f_{n-1, n-1}(x)$ of $X_{n-1, n-1}$ is given by

$$
f_{n-1, n-1}(x)=(n-1)[F(x)]^{n-2} f(x)
$$


And the associated cdf is given by

$$
F_{n-1, n-1}(x)=[F(x)]^{n-1}
$$

Since by hypothesis, we have $F_{Z, W}(x)=F_{n-1, n-1}(x)$, it follows that

$$
\int_{0}^{\infty} n[\bar{F}(x)-\bar{F}(x+y)]^{n-1} f(y) d y=[F(x)]^{n-1} .
$$

Observe that

$$
\int_{0}^{\infty}\{\bar{F}(y)\}^{n-1} f(y) d y=\frac{1}{n}
$$

Hence,

$$
\int_{0}^{\infty}[\bar{F}(x)-\bar{F}(x+y)]^{n-1} f(y) d y=[F(x)]^{n-1} \int_{0}^{\infty}\{\bar{F}(y)\}^{n-1} f(y) d y
$$

i.e.

$$
\begin{gathered}
\int_{0}^{\infty}[\bar{F}(y)-\bar{F}(x+y)]^{n-1} f(y) d y-[F(x)]^{n-1} \int_{0}^{\infty}[\bar{F}(y)]^{n-1} f(y) d y=0, \\
\int_{0}^{\infty}[\bar{F}(y)]^{n-1}\left[\left\{\frac{\bar{F}(y)-\bar{F}(x+y)}{\bar{F}(y)}\right\}^{n-1}-[\bar{F}(x)]^{n-1}\right] f(y) d y=0, \text { and } \\
\int_{0}^{\infty}[\bar{F}(y)]^{n-1}\left[\left\{1-\frac{\bar{F}(x+y)}{\bar{F}(y)}\right\}^{n-1}-[\bar{F}(x)]^{n-1}\right] f(y) d y=0 .
\end{gathered}
$$

On simplification, we obtain

$$
\int_{0}^{\infty}[\bar{F}(y)]^{n-1} G(x, y) f(y) d y=0,
$$

where

$$
G(x, y)=\left\{1-\frac{\bar{F}(x+y)}{\bar{F}(y)}\right\}^{n}-[\bar{F}(x)]^{n}
$$

We have

where $h(x)$ is the hazard rate.

$$
\frac{d}{d y} G(x, y)=n\left\{1-\frac{\bar{F}(x+y)}{\bar{F}(y)}\right\}^{n-1}[h(y)-h(x+y)]
$$

We have $G(x, 0)=0$ and if $h(x)$ is monotonically increasing then for $(2.5)$ to be true, we must have $G(x, y)=0$ for all $x$ and almost all $y$.

Since $G(x, y)=0$ for all $x$ and almost all $y$, then we must have $\frac{d}{d y} G(x, y)=0$. Thus $h(x)$ is constant. Since the hazard rate is a constant, it follows that $X$ is exponential. If $h(x)$ is monotonically decreasing, then a similar arguments leads to the same conclusion.

This completes the proof. 


\section{Conclusion}

In this paper, we have presented two interesting characterizations of the exponential distribution based on order statistic.

\section{Acknowledgements}

A part of this work was done while the second author was visiting Rider University during the summer of 2015. He wishes to acknowledge the warm hospitality received at Rider University and is grateful to the Indian Statistical Institute for granting him leave. The authors thank the referees and the editor for helpful suggestions which improved the presentation of the paper.

\section{References}

Ahsanullah, M., \& Nevzorov, V. B. (2005). Order Statistics.- Examples and Exercises. Nova Science Publications. New York, USA.

Ahsanullah, M., Nevzorov, V. B., \& Shakil, M. (2013). An Introduction to Order Statistics. Atlantis Press. Paris, France.

Arnold, B. C., \& Villasenor, J. A. (2013). Exponential characterizations motivated by the structure of order statistics in sample of size two. Statistics and Probability Letters. 83, 596-601. https://doi.org/10.1016/j.spl.2012.10.028.

Arnold, B. C., Balakrishnan, N., \& Nagaraja, H. N. (1992). A First Course in Order Statistics. John Wiley \& Sons. New York, USA.

David, F. A., \& Nagaraja, H. N. (2009). Order Statistics. Third Edition. Wiley \& Sons. New York, USA.

Ferguson, T. S. (1967). On characterizing distributions by properties of order statistics. Sankhya (Series A). 29, $265-278$.

\section{Copyrights}

Copyright for this article is retained by the author(s), with first publication rights granted to the journal.

This is an open-access article distributed under the terms and conditions of the Creative Commons Attribution license (http://creativecommons.org/licenses/by/4.0/). 